\title{
Simulation of the Plasma Meniscus with and without Space Charge using Triode Extraction System
}

\author{
M.M.Abdel Rahman* and H.El-Khabeary \\ Accelerators \& Ion Sources Department, Nuclear Research Center. Atomic Energy Authority, P.N.13759, Cairo, Egypt.
} (Received on 5 October, 2008)

\begin{abstract}
In this work, simulation of the singly charged argon ion trajectories for a variable plasma meniscus is studied with and without space charge for the triode extraction system by using SIMION 3D (Simulation of Ion Optics in Three Dimensions) version 7 personal computer program. The influence of acceleration voltage applied to the acceleration electrode of the triode extraction system on the shape of the plasma meniscus has been determined. The plasma electrode is set at +5000 volt and the acceleration voltage applied to the acceleration electrode is varied from -5000 volt to +5000 volt. In the most of the concave and convex plasma shapes, ion beam emittance can be calculated by using separate standard deviations of positions and elevations angles. Ion beam emittance as a function of the curvature of the plasma meniscus for different plasma shapes ( flat, concave and convex ) without space charge at acceleration voltage varied from -5000 volt to +5000 volt applied to the acceleration electrode of the triode extraction system has been investigated. The influence of the extraction gap on ion beam emittance for a plasma concave shape of $3.75 \mathrm{~mm}$ without space charge at acceleration voltage, $V_{a c c}=-2000$ volt applied to the acceleration electrode of the triode extraction system has been determined. Also the influence of space charge on ion beam emittance for variable plasma meniscus at acceleration voltage, $V_{a c c}=-2000$ volt applied to the acceleration electrode of the triode extraction system has been studied.
\end{abstract}

Keywords: Ion Beam Emittance; Plasma Meniscus; Space Charge; Ion Trajectories

\section{INTRODUCTION}

The second important step for plasma ion sources, after the production of suitable and high density plasma, is to extract the plasma ions in the form of an ion beam with given kinetic energy. This can be done by using an electrode that is biased at a negative voltage $[1,2]$ with respect to the plasma boundary. The basic problem is to find an optimum geometry for this electrode. Ion beam quality is affected by many factors as the applied extraction voltage, the shape of the emitting plasma surface which may be fixed (field and surface ionization sources) or variable (plasma sources) and on the space charge in the ion beam. The extracted ion beam [3, 4] must be intense with minimum divergence angle and free of aberrations as possible.

The plasma [5] is practically an equipotential and electrically neutral medium. When it comes in contact with an electrode held at a different potential, a plasma sheath is formed and almost the whole potential difference between the plasma and the electrode drops along this sheath. The shape and position of the ion emitting surface (plasma boundary) are affected by the ion density, the extraction voltage applied to the extracting electrode and the geometry of the extraction system. As the potential between the plasma and the electrode may change, the thickness of the sheath varies accordingly. When this potential difference is gradually increased, the plasma boundary retreats until it becomes flat and finally concave at an appropriately high value of the extraction potential.

The extraction of ions from plasma sources has been optimized by computer simulation. Two dimensional (2D) and three dimensional (3D) computer programs are now available for the simulation of ion extraction systems. SIMION 3D version 7.0 computer program [6] is a personnel computer program for designing and analyzing charged particle [7] (ions and electrons) lenses, ion transport systems, various types of

*Electronic address: moustafa82003eyahoo.com mass spectrometers, detector optics, time of flight instruments, ion traps, magnetic sectors and much more. SIMION 3D 7.0 is intended to provide direct and highly interactive methods for simulating a wide variety of general ion optics problems. In general, the fundamental steps for simulating the properties of a model extraction system are to define the physical and electrical boundaries of the electrodes. SIMION defines the ions that make up the beam, selects output data to be recorded and simulates ion accelerated trajectories through the extraction system. Each electrode of the triode extraction system is separately designed using a potential array. Such a potential array is a two or three dimensional array of points, consisting of a collection of equally spaced points forming a rectangular grid. Points in the potential array will be bound within a certain shape creating an electrode or non-electrode. Using a finite difference method, SIMION takes the potentials of the electrode points to calculate the potential at the non-electrode points. Once all three electrodes are designed and defined within a potential array, SIMION solves Laplace's equation:

$$
\nabla^{2} V=0
$$

SIMION uses a highly modified $4^{\text {th }}$ order Runge-kutta technique for modelling the ion trajectories. Ion trajectories are a result of electrostatic and space charge repulsion forces on the basis of the current position and velocity of the ions. These forces are then used to compute the current ion acceleration and used by numerical integration techniques to predict the position and velocity of the ion at the next time step. Electrostatic forces are initially computed in terms of volts per grid unit. As the ion progresses through the potential array it moves from one square of grid points into another. SIMION automatically generates a small 16 point array that represents the current for four grid points and the 12 grid points around it. The values of these grid points are determined by symmetry assumptions and grid point location. The potential at each point is normally calculated via linear interpolation using four grid points bounding the grid square it falls in. When an ion is outside electrostatic instances, SIMION looks both directions along its current trajectory path for the closest electrostatic instance of intersection 
in both directions. If the present ion trajectory intersects electrostatic instances in both directions, SIMION will determine the potentials at the points of intersection and estimate the resulting electrostatic acceleration assuming a linear gradient. In this study, SIMION is used for simulating a plasma meniscus for different plasma shapes (flat, concave and convex) without space charge using the triode extraction system. The influence of different acceleration voltages applied on the acceleration electrode of the triode extraction system on the shape of the plasma meniscus has been determined. Also the effect of space charge on ion beam emittance 8 for variable plasma meniscus using triode extraction system has been investigated.

\section{Construction of the triode extraction system}

Ion beam extraction from ion sources [9] is influenced by many parameters such as electrode geometry, applied extraction voltage, space charge in the extracted beam and finally the shape of the plasma boundary. The construction of the triode extraction system [10] used in this work consists of acceleration / deceleration electrode arrangement with a $5 \mathrm{~mm}$ diameter plasma aperture electrode as shown in Fig. (1). The triode extraction system provides a negative potential with respect to the plasma on axis of the extraction system. A negative potential between the acceleration and the deceleration electrodes creates a barrier for electrons in the extracted beam region to keep them inside the ion beam. These electrons are necessary for the space charge compensation. The plasma electrode terminates the plasma surface at the boundary of the discharge. The downstream side of this electrode must be designed to work as a focusing electrode to provide the proper electric field configuration for optimal ion trajectories.

\section{Calculations for the extraction system for variable plasma meniscus with and without space charge}

In the most of the concave and convex plasma shapes, ion beam emittance can be calculated by using separate standard deviations of positions and elevations angles [11]. For a fixed geometry of the triode extraction system which was described above, the following calculations have been done. After designing the triode extraction system and the operating parameters for simulation of singly charged argon ion trajectories in the extraction region. These parameters as; voltage applied on plasma electrode $V_{\text {plasma }}=+5000 \mathrm{~V}$, voltage applied on the acceleration electrode $\left(V_{\text {accel }}\right)$ was varied from -5000 to $+5000 \mathrm{~V}$. The deceleration electrode was on ground $V_{\text {decel }}=0 \mathrm{~V}$. Flying of ions will be individually and the number of flying ions were 500. First, ion beam emittance for variable plasma meniscus (Flat, concaves and convexes) with different acceleration voltages was investigated with and without space charge. Furthermore, ion beam emittance as a function of the extraction gap for concave plasma meniscus without space charge at assumed acceleration voltage $V_{\text {accel }}=-2000 \mathrm{~V}$ is investigated. Finally, the ion beam emittance as function of the curvature of the variable plasma meniscus at an acceleration voltage $V_{\text {accel }}=-2000 \mathrm{~V}$ is studied for singly charged argon ion trajectories.

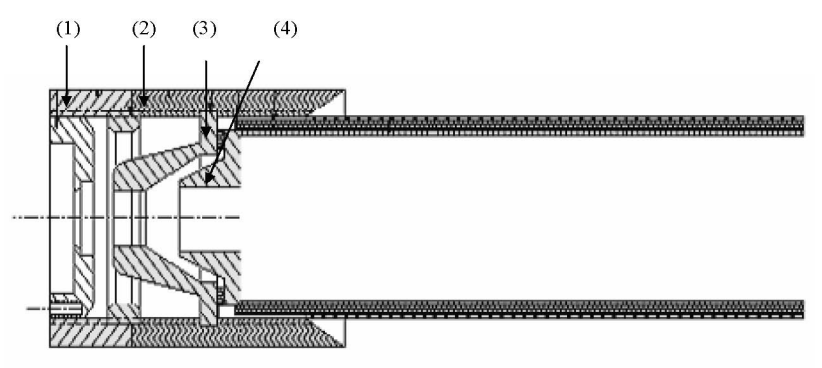

(a)

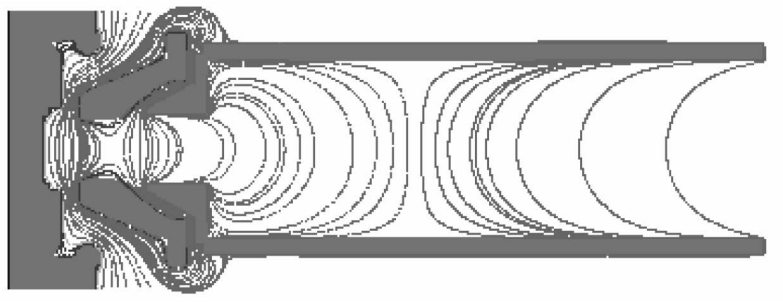

(b)

FIG. 1: Triode extraction system: (a) Triode extraction system designed mechanically: (1) Plasma electrode; (2) Insulator (Macor), (3) Acceleration electrode and (4) Deceleration electrode. (b) Triode extraction system geometry assumed for the SIMION calculations with the contours (see text).

\section{WITHOUT SPACE CHARGE}

2.1 - Flat shape: The influence of the acceleration voltage applied to the acceleration electrode of the triode extraction system on ion beam emittance for flat plasma shape has been investigated as shown in Fig.(2). It is clear that the ion beam emittance increases with increasing the negative voltage applied to the acceleration electrode. The increasing positive application voltage to the acceleration electrode is accompanied by a decrease of the extracted ion beam emittance.

2.2 - Concave shapes: The curvature of the plasma concave shapes of $2.5,2.7,3.5,3.75,4$ and $8.15 \mathrm{~mm}$ were obtained. The influence of the acceleration voltage applied on the acceleration-electrode of the triode extraction system on beam emittance for singly charged argon ion trajectories were investigated (Fig.3). The plasma electrode was at voltage of $+5 k \mathrm{~V}$ and the voltage applied on the acceleration-electrode was varied from -5 to $+5 k V$. For variable concave plasma meniscus, the minimum ion beam emittance was obtained for a curvature of the concave plasma meniscus of $3.75 \mathrm{~mm}$ whiles the maximum at a curvature of $3.5 \mathrm{~mm}$. Applying positive voltage on accelerating-electrode is accompanied by a decrease of the beam emittance. In all curvature cases of the concave plasma meniscus, ion beam emittance was minimum at applied acceleration voltage $V_{\text {accel }}=+5 \mathrm{kV}$, except the curvatures of 3.75 and $4 \mathrm{~mm}$, when the minimum ion beam emittance was obtained at $V_{\text {accel }}=+4 k V$. For a concave plasma meniscus of 2. 7 and $2.5 \mathrm{~mm}$, the decrease of the negative voltage applied is accompanied by an increase of the ion beam emittance till $V_{\text {accel }}=+500 \mathrm{~V}$, and the ion beam emittances decreased by an increase of the positive voltage. 


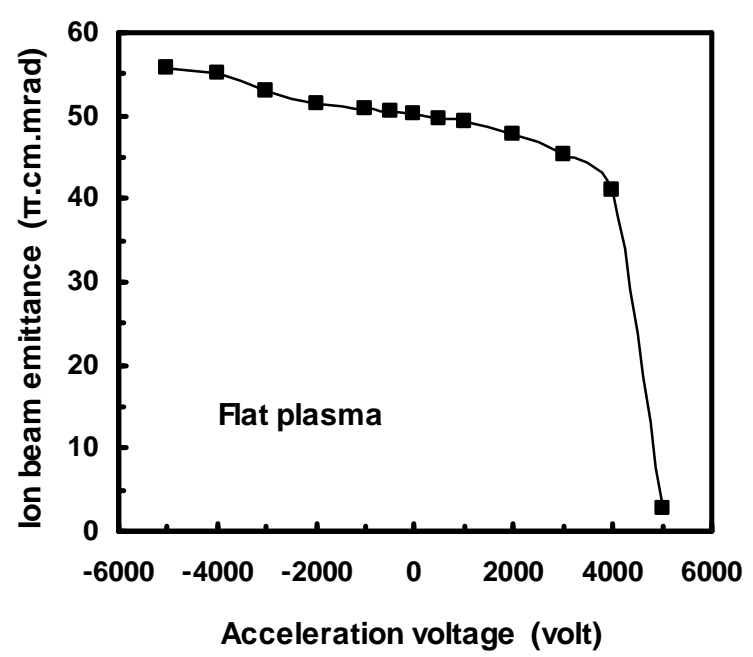

FIG. 2: Influence of the acceleration voltage on ion beam emittance for flat plasma shape without space charge.

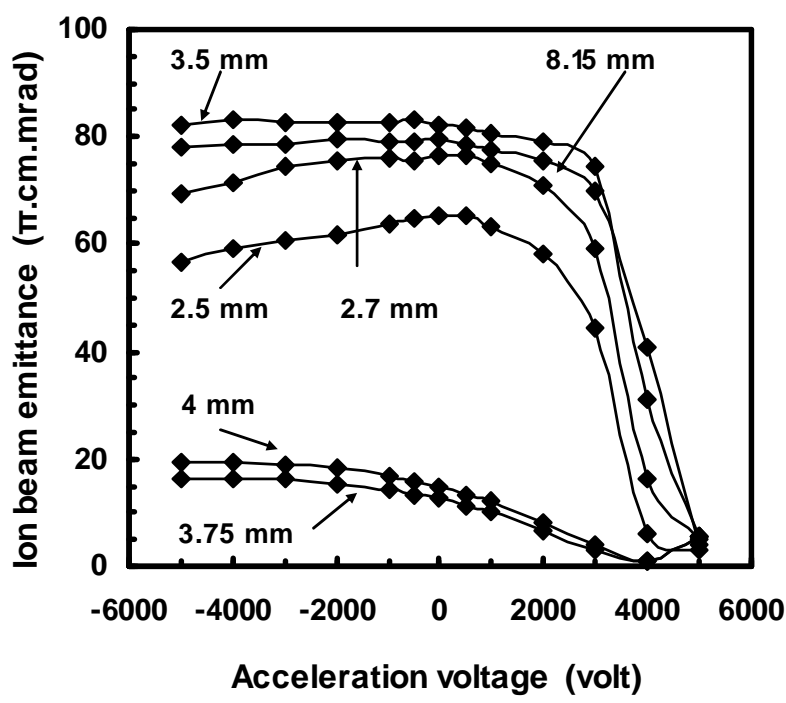

FIG. 3: Influence of the acceleration voltage on ion beam emittance for various curvatures of the concave plasma shapes without space charge.

2.3 - Convex shapes: The same curvatures of the convex plasma meniscus were obtained as in plasma concave shapes. Also, influence of the acceleration voltage on ion beam emittance for singly charged argon ion trajectories was investigated (Fig.4). For variable convex plasma meniscus, the minimum ion beam emittance was obtained for a curvature of 2.7 $\mathrm{mm}$. Maximum ion beam emittance was obtained at curvature of the convex plasma meniscus of $3.5 \mathrm{~mm}$. As in a concave plasma meniscus shapes, curvatures of the convex plasma meniscus of $3.5 \mathrm{~mm}$, the decrease of negative voltage applied on acceleration-deceleration electrode is accompanied by the decrease of the ion beam emittance, also, for

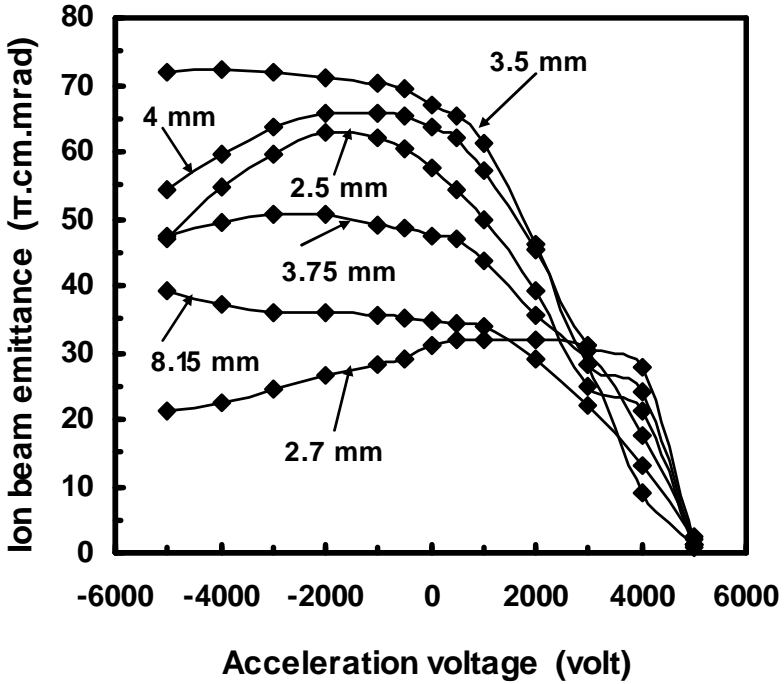

FIG. 4: Influence of the acceleration voltage on ion beam emittance for various curvatures of convex plasma shapes without space charge.

positive voltage; an increase of the voltage is accompanied by a decrease of ion beam emittance. With respect to the curvature of the convex plasma meniscus of $3.75 \mathrm{~mm}$, the decrease of negative voltage is accompanied by an increase of beam emittance till $V_{\text {accel }}=0 \mathrm{~V}$, ion beam emittance started to decrease by an increase of the positive voltage. For the curvature of the convex plasma meniscus of $2.5 \mathrm{~mm}$, the decrease of the voltage is accompanied by slightly increase of the ion beam emittance till $V_{\text {accel }}=-1000 \mathrm{~V}$, ion beam emittance decreased till $V_{\text {accel }}=-500 \mathrm{~V}$. When there was no voltage applied $V_{\text {accel }}=0 \mathrm{~V}$, ion beam emittance increased then decreased by an increase of the positive voltage till $V_{\text {accel }}=+3000 \mathrm{~V}$, there was a sharp decrease of the ion beam emittance by an increase of the positive voltage. For curvature of the convex plasma meniscus of $8.15 \mathrm{~mm}$, a decrease of the applied voltage is accompanied by the decrease of the ion beam emittance till $V_{\text {accel }}=+3000 \mathrm{~V}$, the ion beam emittance increased, then sharply decreased. Minimum ion beam emittance obtained of curvature $2.7 \mathrm{~mm}$ was increased by the decrease of the voltage till $V_{\text {accel }}=+1000 \mathrm{~V}$, then decreased by an increase of the voltage. In all curvature cases of the convex plasma meniscus, beam emittance was minimum at applied acceleration voltage $V_{\text {accel }}=+5 \mathrm{kV}$ (Fig.4). The ion beam emittance as a function of the curvature of the variable plasma meniscus at an acceleration voltage $V_{\text {accel }}=-2 \mathrm{kV}$ was studied for singly charged argon ion trajectories. The curvatures of the concave plasma meniscus of 2.5, 2.7, 3.5, 3.75, 4 and $8.15 \mathrm{~mm}$ were obtained. As for concave plasma meniscus, the curvatures of the convex plasma meniscus were also obtained. At an acceleration voltage applied on the acceleration-electrode of the triode extraction system $V_{\text {accel }}=-2 k V$, beam emittance was studied as function of the curvature of these obtained variable plasma meniscus. The ion beam emittance for flat plasma was not considered. The minimum ion beam emittance was found at curvature of concave plasma meniscus of $3.75 \mathrm{~mm}$ and a curvature of convex plasma meniscus of $2.7 \mathrm{~mm}$ (Fig.5).

The variation of the distance between the plasma elec- 


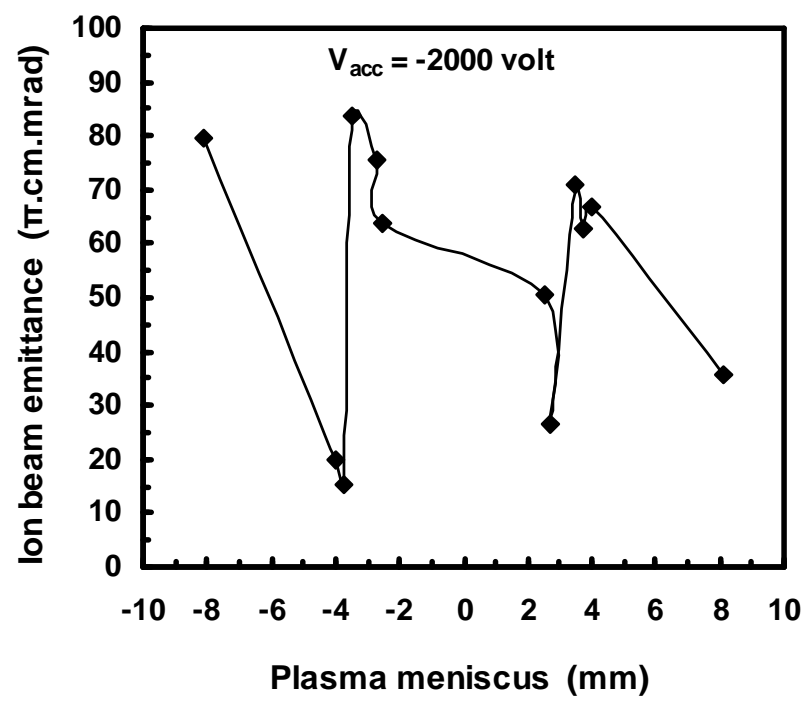

FIG. 5: Ion beam emittance as a function of the curvatures of plasma meniscus at $V_{a c c}=-2000$ volt.

trode and the acceleration-electrode (extraction gap) was investigated without space charge on the curvature of concave plasma meniscus of $3.75 \mathrm{~mm}$ at acceleration voltage applied on the acceleration- electrode $V_{\text {accel }}=-2 k V$ (Fig.6). This curvature of the concave plasma meniscus was chosen because the ion beam emittance was minimum and at this curvature the plasma boundary has an optimum curvature. It was found that the minimum ion beam emittance was obtained at extraction gap of $15.5 \mathrm{~mm}$. For ion beam extraction, the current, the perveance is given by [12] :

$$
P=\frac{I_{i}}{V^{3 / 2}} \times\left(\frac{A}{z}\right)^{1 / 2}=\frac{4 \varepsilon_{0}}{9} \sqrt{\frac{2 q}{m}} \frac{A}{d^{2}}
$$

where $A$ is the emitting area, $z$ is the charge, $I_{i}$ total ion beam current, $\varepsilon_{0}$ is the free space permittivity, $d$ is the extraction gap width and $V$ is the acceleration voltage.

\section{WITH SPACE CHARGE}

Ion beams which propagate through a given beam line are subject to blow up because of the tendency of like charged (positive) ions within the ion beam to mutually repel each other (space charge effect). The influence of ion space charge is disadvantage for both the quality and the intensity of extracted ion beams. In order to reduce this problem, space charge neutralization (compensation) has to be used.

In the presence of space charge, the electric field acting on an ion beam is $[13,14]$ :

$$
E_{r}=\frac{q}{2 \pi \varepsilon_{0} r}=\frac{I_{0}}{2 \pi \varepsilon_{0} \nu_{0} r},
$$

where $q$ is the charge of the beam per unit length within radius $r$ and $q=\frac{I_{0}}{v_{0}}$ where $I_{0}$ is the total beam current, $v_{0}$ is the axial ion velocity. SIMION program supports three estimates of charge repulsion: beam, columbic and factor repul-

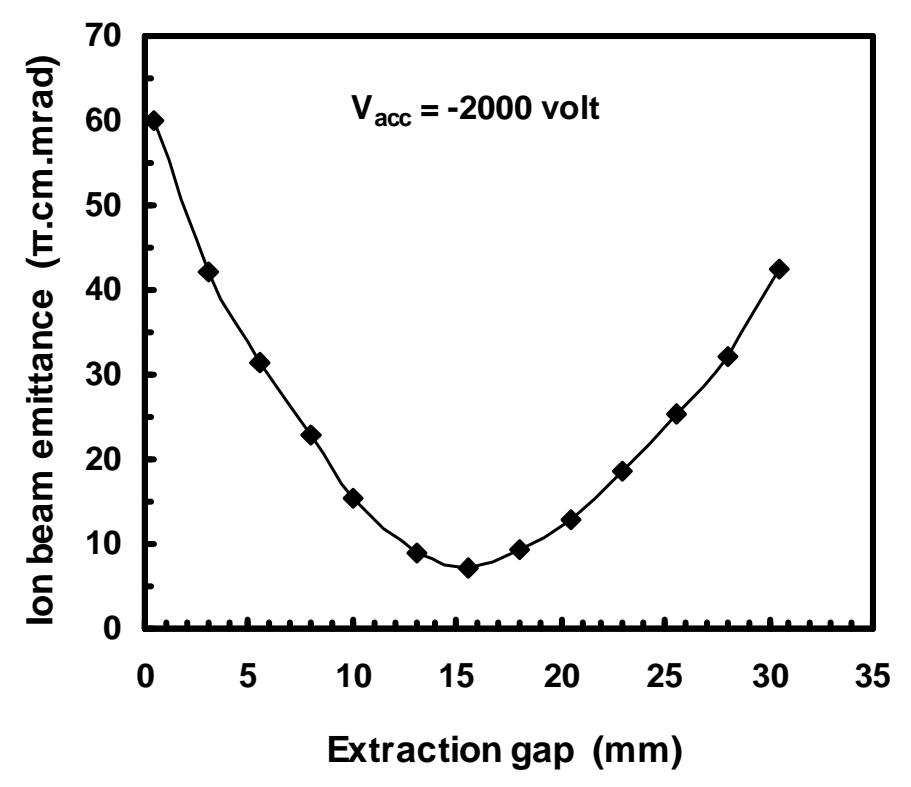

FIG. 6: Influence of the extraction gap on ion beam emittance for a concave plasma shape of $3.75 \mathrm{~mm}$.

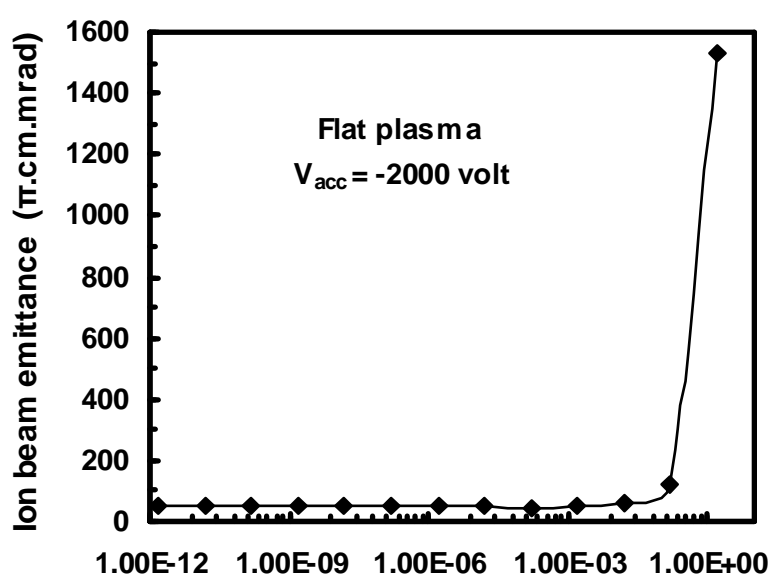

Current (A)

FIG. 7: nfluence of space charge on ion beam emittance for flat plasma shape at $V_{a c c}=-2000$ volt

sion. In general, charge repulsion estimations involve determining the forces between the current ion in question and all other currently flying ions. In this work, simulation of the singly charged argon ion trajectories for a variable meniscus shape of the plasma is studied with space charge effect by using columbic repulsion. Figure (7) shows the influence of the space charge on ion beam emittance at acceleration voltage $V_{a c c}=-2000$ volt applied to the acceleration electrode of the triode extraction system for flat plasma. It is clear that for flat plasma, space charge has a slightly effect on the extracted ion beam emittance and started to have a large effect at current higher than $10^{-1} A$. 


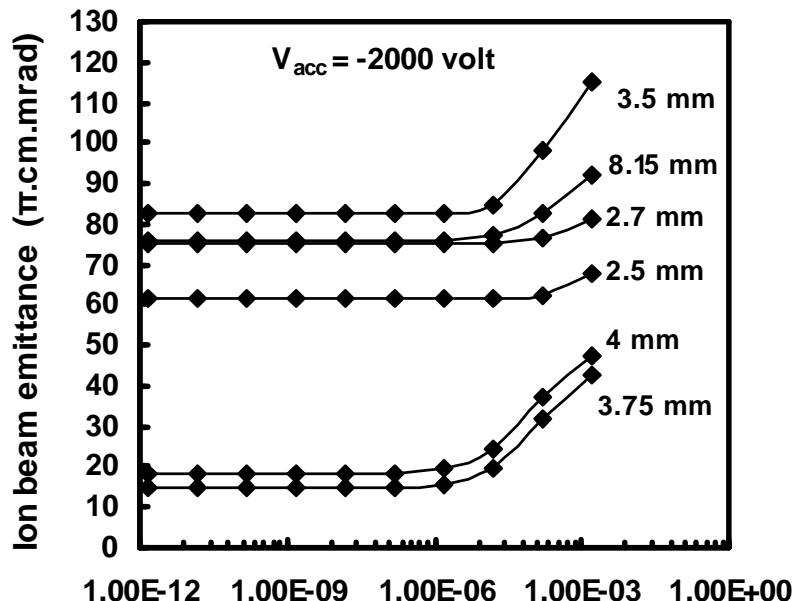

Current (A)

FIG. 8: Influence of space charge on ion beam emittance for different curvatures of concave plasma shapes

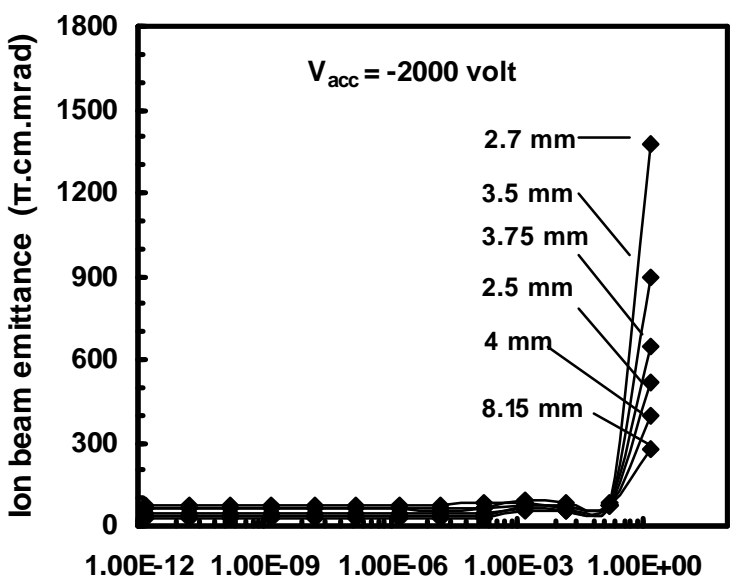

Current (A)

FIG. 9: Influence of space charge on ion beam emittance for different curvatures of convex plasma shapes

The influence of space charge on the extracted ion beam emittance for a variable curvatures of concave plasma meniscus at acceleration voltage, $V_{a c c}=-2000$ volt applied to the acceleration electrode of the triode extraction system has been studied as shown in Fig.(8). It is found that the space charge has a large effect on the extracted ion beam emittance for different curvatures of concave plasma shapes at current higher than $10^{-4} \mathrm{~A}$. Figure (9) shows the influence of the space charge on the extracted ion beam emittance for a variable curvatures of convex plasma meniscus at acceleration voltage, $V_{a c c}=-2000$ volt applied to the acceleration electrode of the triode extraction system. It is obvious that no effect for space charge on the extracted ion beam emittance, till the current of $10^{-1} A$, then the extracted ion beam emittance increased.

\section{CONCLUSION}

The simulation of singly charged argon ion trajectories for variable curvatures of plasma meniscus is studied with and without space charge for triode extraction system using SIMION 3D version 7.0 personal computer program. The influence of the acceleration voltage ( varied from -5000 volt to +5000 volt ) applied to the acceleration electrode of the triode extraction system on the extracted ion beam emittance has been investigated. It can be concluded that without space charge at acceleration voltage $V_{a c c}=-2000$ volt applied to the acceleration electrode of the triode extraction system, the minimum ion beam emittance is found at a curvature of concave plasma shape equal to $3.75 \mathrm{~mm}$ and a curvature of convex plasma shape equal to $2.7 \mathrm{~mm}$. Also the influence of the extraction gap on the extracted ion beam emittance without space charge at acceleration voltage, $V_{a c c}=-2000$ volt applied to the acceleration electrode for a curvature of concave plasma shape equal to $3.75 \mathrm{~mm}$ has been determined. It is found that the minimum ion beam emittance is obtained at extraction gap equal to $15.5 \mathrm{~mm}$. Also the effect of space charge on the extracted ion beam emittance for variable plasma meniscus at acceleration voltage, $V_{a c c}=-2000$ volt applied to the acceleration electrode of the triode extraction system has been studied. It is found that the space charge has a large effect on the extracted ion beam emittance for a different concave plasma shapes at current higher than $10^{-6} \mathrm{~A}$. For flat plasma, the space charge has a slightly effect on the extracted ion beam emittance and started to have a large effect at current higher than $10^{-1} \mathrm{~A}$. But for different convex plasma shapes, there is no effect of the space charge on the extracted ion beam emittance till the current of $10^{-1} \mathrm{~A}$ when the extracted ion beam emittance increased. Also from this study, it can be concluded that the construction of the triode extraction system with extraction gap equal $15.5 \mathrm{~mm}$ and acceleration voltage, $V_{a c c}=-2000$ volt applied to the acceleration electrode is suitable for optimum extraction of ion beam from variable plasma meniscus.
[1] A.G.Drentje; Rev. Sci. Instrum., 75, 1399 (2004).

[2] M.Leitner, D.Wutte, J.Brandstotter, F.Aumayr and HP.Winter; Rev. Sci. Instrum., 65, 1091 (1994).

[3] J.B.Rosenzweig; Fundamentals of Beam Physics, Oxford University Press, England (2003).

[4] L.A.Giannuzzi and F.A.Stevie; Introduction to Focused Ion Beams, Springer, USA (2005).
[5] T.J.M.Boyd; The Physics of Plasmas, Cambridge University Press, England (2003).

[6] D.A.Dahl, SIMION 3D Version 7.0 User's Manual INEEL95/0403, Idaho National Engineering and Environmental Laboratory, ID 83415 (2000).

[7] J.Orloff; Handbook of Charged Particle Optics', CRC Press, Boca Raton, New York, 275 (1997). 
[8] K.Floettmann; Physical Review Special Topics; 6, 1 (2003).

[9] B.H.Wolf; Handbook of Ion Sources, CRC Press, Boca Raton, New York (1995).

[10] M.M.Abdelrahman; Investigation of a $14.5 \mathrm{GHz}$ ECR Ion Source for Experiments on Collisions with Slow Multicharged Ions, Ph.D. Thesis, Faculty of Phys., Vienna University of Technology, Vienna (2004).

[11] M.M. Abdelrahman, Two and three electrostatic lens systems for focusing of charged particles, accepted for publication in Arab journal of nuclear sciences and applications (2008).

[12] A. Spetier, Focusing of Charged Particles, Vol. II, Academic press, New York and London (1967).

[13] R.G. Wilson and G.R. Brewer; Ion Beams with Applications to Ion Implantation, John Wiley \& New York (1973)

[14] H. Wituschek et al.; Rev. Sci. Instrum. 63; 2785 (1992). 